Prospects for Electron Imaging with Ultrafast Time Resolution

M. R. Armstrong, B. W. Reed, B. R. Torralva, N.

D. Browning

February 15, 2007

Applied Physics Letters 
This document was prepared as an account of work sponsored by an agency of the United States Government. Neither the United States Government nor the University of California nor any of their employees, makes any warranty, express or implied, or assumes any legal liability or responsibility for the accuracy, completeness, or usefulness of any information, apparatus, product, or process disclosed, or represents that its use would not infringe privately owned rights. Reference herein to any specific commercial product, process, or service by trade name, trademark, manufacturer, or otherwise, does not necessarily constitute or imply its endorsement, recommendation, or favoring by the United States Government or the University of California. The views and opinions of authors expressed herein do not necessarily state or reflect those of the United States Government or the University of California, and shall not be used for advertising or product endorsement purposes. 


\title{
Prospects for Electron Imaging with Ultrafast Time Resolution
}

\author{
Michael R. Armstrong, Bryan W. Reed, and Ben R. Torralva
}

Materials Science and Technology Division, Chemistry and Materials Science Directorate, Lawrence Livermore National Laboratory, P.O. Box 808; L-356, Livermore, CA 94550

\section{Nigel D. Browning}

Department of Chemical Engineering and Materials Science, University of California-Davis, One Shields Ave, Davis, CA 95616

\begin{abstract}
Many pivotal aspects of material science, biomechanics, and chemistry would benefit from nanometer imaging with ultrafast time resolution. Here we demonstrate the feasibility of shortpulse electron imaging with 10 nanometer/10 picosecond spatio-temporal resolution, sufficient to characterize phenomena that propagate at the speed of sound in materials (1-10 kilometer/second) without smearing. We outline resolution-degrading effects that occur at high current density followed by strategies to mitigate these effects. Finally, we present a model electron imaging system that achieves 10 nanometer/10 picosecond spatio-temporal resolution.
\end{abstract}


Due to the speed limitations of electronics, typically ultrafast time domain experiments (including imaging) require "flash" illumination of the sample in analogy with time-resolved pump-probe optical spectroscopy. The time resolution for this technique is determined by the duration of the pump and probe pulses - the pulse duration must be shorter than the time scale of dynamics of interest. The analogous experiment with electron imaging (or diffraction) involves initiation of sample dynamics followed by sample illumination with an electron probe pulse. Scattered electrons from the probe pulse may be projected via electron optics onto a 2D detector to obtain a magnified image (or diffraction pattern) of the sample, as shown in Fig. 1. For single shot imaging, the image must be obtained with a single electron probe pulse after a single pump pulse.

Pump-probe x-ray and electron diffraction techniques have recently been employed in the investigation of phenomena ranging from biomolecule structural dynamics to ultrafast melting [19]. Diffraction experiments have a great advantage over spectroscopy, as diffraction directly probes atomic positions. Typically, diffraction experiments can achieve atomic (Angstrom scale) spatial resolution even with ultrafast time resolution, while static imaging with electrons can achieve subAngstrom resolution.[10,11] Although such results provide invaluable insight into ultrafast processes on atomic length scales, diffraction is significantly limited in field of view, requires a sample with a periodic character, and is inherently an ensemble measurement $[6,12]$. Yet, material dynamics often occur in contexts which are inherently non-periodic with a larger field of view [13]. Diffractive imaging techniques are being developed to address these issues [6,14-16], but direct imaging is ideally suited to characterize such systems and electron imaging has played a pivotal role in the discovery of phenomena that are difficult to observe with diffraction $[17,18]$. Also, the 
dynamics of phenomena at the 10 nanometer scale are often unique and non-reversible. At ultrafast time scales, such data may only be obtained with a single shot probe. Here we define ultrafast as sufficient time resolution to characterize effects that propagate at the speed of sound in the sample without smearing at the achievable spatial resolution.

The primary problem with obtaining high temporal and spatial resolution with electrons in this scheme involves the degradation of resolution through Coulomb interactions within the probe pulse. Although ultrafast stroboscopic imaging avoids space charge issues [19], in general imaging is most useful for the observation of stochastic, irreversible processes which are difficult to consistently prepare. Yet, ultrafast stroboscopic imaging requires a sample which can rapidly, identically refresh for up to $10^{7}$ experiments to built up an image. Here we outline the relevant issues for single shot ultrafast imaging and present a model electron optical system which obtains high spatial resolution ultrafast images.

To obtain a high quality image, a minimum scattered particle fluence (i.e. detected particles scattered per unit sample area) is required to achieve a given spatial resolution via shot noise statistics. A rough constraint for distinguishing features in an image is given by requiring the shot noise in a single pixel to be less than the contrast in the image by a quality factor - the Rose criterion [20],

$$
\text { Shot noise } \leq \frac{\text { contrast }}{\# \text { of gray scale levels }}
$$

Typically, the number of gray scale levels (the contrast divided by the noise) must be around 3-5 for minimum acceptable image quality. Shot noise is given by

$$
\text { Shot noise }=\frac{1}{\sqrt{N}}
$$


where $\mathrm{N}=$ the number of detected particles per pixel. So, for $10^{6}$ detected particles scattered from a sample with 50\% contrast in a shot noise limited imaging system, the noise would have to be below $10 \%$ by eq. 1 (assuming 5 gray scale levels). By eq. 2 , this requires a pixel size that is sufficiently large to detect an average of 100 particles per pixel, giving $10^{4}$ pixels for $10^{6}$ total detected electrons across the field of view - a 100x100 image. In the case of a $1 \mu \mathrm{m}^{2}$ illuminated sample region, this implies a shot noise limited resolution of $10 \mathrm{~nm}$. For imaging systems which use direct current to illuminate the sample, the peak current can be arbitrarily small (i.e. low current with long integration time), effectively eliminating Coulomb interactions between electrons. For ultrafast imaging, the added requirement to obtain high temporal resolution in a single shot constrains the peak current to be much higher. In particular, an electron pulse of $10 \mathrm{ps}$ duration containing $10^{6}$ electrons has a peak current of approximately $20 \mathrm{~mA}$, many orders of magnitude larger than the current found in standard transmission electron microscopy $(\sim \mathrm{nA})$. In this regime Coulomb effects are significant and must be considered in the electron optical design of the imaging system.

The effects of Coulomb interactions in an electron pulse can be broadly divided into two categories: large scale, or "global" space charge (GSC) effects, and stochastic scattering between individual electrons. GSC effects are relatively straightforward: on large scales a uniform distribution of charge remains uniform as it propagates [21, 22]. From an electron optical perspective, uniform global space charge behaves precisely as a negative lens and the effects of global space charge can be corrected to first order by electron optics. In a real imaging system, the electron pulse will not be uniform, and global space charge may result in higher order aberrated distortions of the electron pulse, potentially requiring the use of aberration corrected electron lenses [23]. Global space charge will not significantly degrade the resolution with proper design of the 
imaging system, but random smaller scale electron interactions still limit the achievable resolution at high current density.

Stochastic scattering fundamentally limits the achievable resolution through random electronelectron scattering, which results in irreversible loss (blurring) of image information as the pulse propagates [24, 25]. Essentially, image information encoded in electron trajectories (after being scattered from the sample) is lost via thermal equilibration. This effect has a significant impact on high current charged particle optical systems and has been modeled within this context. [24-30] Typically, the degree of resolution degradation is characterized by a blur profile - a histogram of the deviation (in the image plane) between image forming electrons which are ray traced through the optical system and electrons whose trajectories are perturbed by Coulomb interactions. This procedure gives a blur profile, which is similar to the point spread function used as a transfer function in traditional optics and may be used to estimate the optimum resolution of the imaging system. Since blur cannot be completely eliminated, it must be mitigated by reducing the current density through instrument design.

We explored two strategies to reduce the effects of stochastic blur: the use of relativistic pulses and annular dark field imaging. For picosecond duration pulses of sufficient charge to image in a single shot, the use of relativistic electron energy is necessary to mitigate stochastic blur and maintain an ultrashort pulse duration over relatively long propagation lengths [31]. Although blur is significantly reduced at high electron energy, our simulations indicate that even for short propagation lengths $(\sim 1 \mathrm{~cm})$ at $5 \mathrm{MeV}$ electron energy, $10^{6}$ electrons in 10 ps gives roughly $\sim 10 \mathrm{~nm}$ of blur. An actual imaging system would involve much longer propagation lengths (resulting in 
higher blur), so this strategy alone is insufficient to achieve $10 \mathrm{~nm} / 10 \mathrm{ps}$ spatial-temporal resolution. In principle, arbitrarily high energy pulses could be used to reduce blur indefinitely, but this is practically limited by the strength of electron lenses.

To achieve high resolution, it is also necessary to reduce the average current density. One way to accomplish this is annular dark field imaging (ADF). In ADF, the image is formed by scattered electrons, as shown in Fig. 1. Light regions in the image correspond to regions of strong scattering in the sample [32]. ADF is advantageous because it removes electrons that would only contribute to a bright background without carrying image information about the sample. Also, by only accepting electrons that are scattered to large angles, the average current density in the optical system can be significantly reduced. Finally, since only scattered electrons form the image in ADF, the total image contrast is $100 \%$, which decreases the minimum required fluence from the Rose criterion.

To demonstrate the feasibility of high spatio-temporal electron imaging, we modeled an imaging system with a $5 \mathrm{MeV}$ illumination electron pulse focused to a 1 micron diameter spot on a copper sample with sufficiently small divergence for unscattered electrons to be blocked after the sample, allowing scattered electrons to continue through the imaging system (as shown in Fig. 1). We used electron differential scattering cross sections calculated by the ELSEPA computer program [33] assuming a Fermi-Thomas model of the nucleus for a solid copper sample. The simulated electron optical system consists of thin lenses with focal lengths of $1.0 \mathrm{~cm}$ (objective), $0.74 \mathrm{~cm}\left(1^{\text {st }}\right.$ intermediate), and $1.6 \mathrm{~cm} \mathrm{(2} 2^{\text {nd }}$ intermediate), with magnifications of $6.3 \mathrm{x}, 47 \mathrm{x}$, and $18 \mathrm{x}$, respectively, for a total magnification of $\sim 5300 x$. The dynamics of the electron pulse were solved by computation of Coulomb forces without approximation and propagation of point particles via a 
symplectic leap frog algorithm, typically performed on between 10-40 nodes of a Unix cluster at LLNL for $2 \times 10^{4}$ electrons at a density consistent with $10^{6}$ electrons in a 10 ps duration pulse. There was not a strong dependence on the number of electrons at the same density for $>2 \times 10^{4}$ electrons, indicating that edge effects are negligible in this model. The stochastic blur was calculated for three stages of magnification since blur did not increase significantly thereafter, where the current density is smaller and blur is less significant on the scale of the magnified image. The blur profile of this imaging system with a Gaussian profile fit is shown in Fig. 2. As illustrated in the figure, these simulations indicate it is possible to obtain less than $10 \mathrm{~nm}$ of stochastic blur with a 10 ps duration pulse, assuming $10^{6}$ electrons scattered to greater than 5 mrad half angle. Assuming single electron sensitive detection and $10^{6}$ image forming electrons, the illumination pulse need not have more than a few $\mathrm{pC}$ of charge to obtain $10 \mathrm{~nm}$ spatial resolution with a pulse that is short enough to obtain $10 \mathrm{ps}$ time resolution. A more intuitive illustration of the spatial resolution is shown in Fig. 3, which shows a CdTe tetrapod, CdTe nanoparticle and gold nanoparticle imaged using high angle ADF imaging [32] (figure 3a) and a simulation of this image assuming the blur and noise limitations of the model (figure 3b). The object image was used as presented in ref. 32. Figure $3 \mathrm{~b}$ was constructed by assuming a random, spatially uniform distribution of electrons are scattered in proportion to the image intensity of figure $3 \mathrm{a}$, with a scattered, detected fluence sufficient to satisfy the Rose criterion $\left(10^{6} \mathrm{e} / \mu \mathrm{m}^{2}\right)$ where the intensity of figure $3 \mathrm{a}$ is $100 \%$. To generate figure $3 \mathrm{~b}$, these "scattered" electrons are then binned into $5 \times 5 \mathrm{~nm}^{2}$ pixels (on the scale of figure 3a) after randomly smearing their positions using the distribution of figure 2 to account for stochastic scattering. Fine structure at the $10 \mathrm{~nm}$ scale (such as the boundary of the gold nanoparticle) is captured in this image, demonstrating the possibility of high resolution imaging using an ultrashort pulse of electrons. Without relativistic electrons and ADF imaging with 
$10^{6}$ scattered electrons, stochastic scattering would randomly distribute these image electrons over a region that is larger than the field of view, completely washing out image information on the scale of fig. 3a.

Given the feasibility of this method in principle, two technologies require further development to realize such an instrument in practice: high beam quality short-pulse electron guns and powerful magnetic lenses systems capable of operating in the several-MeV range. To obtain a sufficiently small illumination area and beam divergence, the transverse geometric beam emittance must be on the order of some nm. Furthermore, standard transmission electron microscopy is limited by chromatic aberration, which constrains the variation in electron energy to less than $10^{-5}$ for Angstrom scale resolution. Although typical radio frequency (RF) accelerators can achieve transverse normalized emittance around $1 \mu \mathrm{m}$ with $0.1-1.0 \%$ energy variation, more work is required to achieve the generation of high quality ultrashort electron beams $[21,34,35]$. Furthermore, we have found that stochastic blur is mitigated by reducing the distance to achieve high magnification, which implies very strong lenses with focal lengths of order $1 \mathrm{~cm}$ or less for 5 $\mathrm{MeV}$ electrons. Such short focal lengths at $\mathrm{MeV}$ energy will require powerful, possibly superconducting lenses [36, 37].

In conclusion, the optimum resolution in electron imaging is constrained at low current density by signal to noise requirements and at high density by stochastic scattering of electrons. Simulations of stochastic scattering in a model electron imaging system indicate that it is possible to obtain $10 \mathrm{~nm}$ spatial and 10 ps temporal resolution by imaging with $5 \mathrm{MeV}$ electrons and the use of annular dark field imaging. Such an imaging system would require improvements in the beam quality available 
from short-pulse $\mathrm{MeV}$ electron sources and the development of high power electron lenses, and would open a horizon in the study of ultrafast material dynamics.

This work was performed under the auspices of the U.S. Department of Energy by the University of California, Lawrence Livermore National Laboratory under Contract W-7405-Eng-48. We would also like to acknowledge helpful comments from Wayne E. King. 


\section{References}

[1] A. H. Zewail, Philos T Roy Soc A 363, 315 (2005).

[2] B. J. Siwick, J. R. Dwyer, R. E. Jordan, and R. J. D. Miller, Science 302, 1382 (2003).

[3] J. M. Cao, H. Ihee, and A. H. Zewail, P Natl Acad Sci USA 96, 338 (1999).

[4] K. J. Gaffney, A. M. Lindenberg, J. Larsson, K. Sokolowski-Tinten, C. Blome, O. Synnergren, J. Sheppard, C. Caleman, A. G. MacPhee, D. Weinstein, D. P. Lowney, T. Allison, T. Matthews, R. W. Falcone, A. L. Cavalieri, D. M. Fritz, S. H. Lee, P. H. Bucksbaum, D. A. Reis, J. Rudati, A. T. Macrander, P. H. Fuoss, C. C. Kao, D. P. Siddons, R. Pahl, K. Moffat, J. Als-Nielsen, S. Duesterer, R. Ischebeck, H. Schlarb, H. SchulteSchrepping, J. Schneider, D. von der Linde, O. Hignette, F. Sette, H. N. Chapman, R. W. Lee, T. N. Hansen, J. S. Wark, M. Bergh, G. Huldt, D. van der Spoel, N. Timneanu, J. Hajdu, R. A. Akre, E. Bong, P. Krejcik, J. Arthur, S. Brennan, K. Luening, and J. B. Hastings, Phys Rev Lett 95, 125701 (2005).

[5] F. Schotte, M. H. Lim, T. A. Jackson, A. V. Smirnov, J. Soman, J. S. Olson, G. N. Phillips, M. Wulff, and P. A. Anfinrud, Science 300, 1944 (2003).

[6] R. Neutze, R. Wouts, D. van der Spoel, E. Weckert, and J. Hajdu, Nature 406, 752 (2000).

[7] C. Y. Ruan, V. A. Lobastov, F. Vigliotti, S. Y. Chen, and A. H. Zewail, Science 304, 80 (2004).

[8] A. Grigoriev, D. H. Do, D. M. Kim, C. B. Eom, B. Adams, E. M. Dufresne, and P. G. Evans, Phys Rev Lett 96, 187601 (2006).

[9] J. B. Hastings, R. M. Rudakov, D. H. Dowell, J. F. Schmerge, J. D. Cardoza, J. M. Castro, S. M. Gierman, H. Loos, and P. M. Weber, Appl Phys Lett 89, 184109 (2006).

[10] H. Rose, Optic 85, 19 (1990). 
[11] B. Freitag, S. Kujawa, P. M. Mul, J. Ringnalda, and P. C. Tiemeijer, Ultramicroscopy 102, 209 (2005).

[12] J. M. Zuo, I. Vartanyants, M. Gao, R. Zhang, L. A. Nagahara, Science 300, 1419 (2003).

[13] A. M. Minor, E. T. Lilleodden, E. A. Stach, and J. W. Morris, J Mater Res 19, 176 (2004).

[14] J. C. H. Spence, U. Weierstall, and M. Howells, Ultramicroscopy 101, 149 (2004).

[15] H. N. Chapman, A. Barty, S. Marchesini, A. Noy, S. R. Hau-Riege, C. Cui, M. R. Howells, R. Rosen, H. He, J. C. H. Spence, U. Weierstall, T. Beetz, C. Jacobsen, and D. Shapiro, J Opt Soc Am A 23, 1179 (2006).

[16] G. J. Williams, H. M. Quiney, B. B. Dhal, C. Q. Tran, K. A. Nugent, A. G. Peele, D. Paterson, and M. D. de Jonge, Phys Rev Lett 97, 025506 (2006).

[17] P. B. Hirsch, R. W. Horne, and M. J. Whelan, Philos Mag 1, 677 (1956).

[18] S. Iijima, Nature 354, 56 (1991).

[19] V. A. Lobastov, R. Srinivasan, and A. H. Zewail, P Natl Acad Sci USA 102, 7069 (2005).

[20] A. Rose, in Advances in Electronics and Electron Physics, Vol. I (Academic Press, New York, 1948), p. 131.

[21] O. J. Luiten, S. B. van der Geer, M. J. de Loos, F. B. Kiewiet, and M. J. van der Wiel, Phys Rev Lett 93, 094802 (2004).

[22] B. W. Reed, J. Appl Phys 100, 034916 (2006).

[23] E. M. James, N. D. Browning, A. W. Nicholls, M. Kawasaki, Y. Xin, and S. Stemmer, J Electron Microsc 47, 561 (1998).

[24] A. Weidenhausen, R. Spehr, and H. Rose, Optic 69, 126 (1985).

[25] G. H. Jansen, Coulomb Interactions in Particle Beams (Academic, San Diego, CA, 1990)Vol. 21, p. 546. 
[26] G. H. Jansen, J Appl Phys 84, 4549 (1998).

[27] J. A. Liddle, M. I. Blakey, K. Bolan, R. C. Farrow, G. M. Gallatin, R. Kasica, V. Katsap, C. S. Knurek, J. Li, M. Mkrtchyan, A. E. Novembre, L. Ocola, P. A. Orphanos, M. L. Peabody, S. T. Stanton, K. Teffeau, W. K. Waskiewicz, and E. Munro, J Vac Sci Technol B 19, 476 (2001).

[28] M. Mkrtchyan, J. A. Liddle, S. D. Berger, L. R. Harriott, J. M. Gibson, and A. M. Schwartz, J Appl Phys 78, 6888 (1995).

[29] P. Kruit, and G. H. Jansen, in Handbook of Charged Particle Optics, edited by J. Orloff (CRC Press, Boca Raton, 1997), pp. 275.

[30] One of Jansen's papers [26] contains an inconsistency in one figure. Jansen's fits give a curve that is significantly different than the curve shown in Fig. 8 of that work.

[31] B. J. Siwick, J. R. Dwyer, R. E. Jordan, and R. J. D. Miller, J Appl Phys 92, 1643 (2002).

[32] S. Bals, B. Kabius, M. Haider, V. Radmilovic, and C. Kisielowski, Solid State Commun 130, 675 (2004).

[33] F. Salvat, A. Joablonski, and C. Powell, Comput Phys Commun 165, 157 (2005).

[34] J. W. Lewellen, in LINAC 2004, Lubeck, Germany (2004).

[35] B. J. Claessens, S. B. van der Geer, G. Taban, E. J. D. Vredenbregt, and O. J. Luiten, Phys Rev Lett 95, 164801 (2005).

[36] H. K. Ito and L. Grodzins, Bull Am Phys Soc 24, 595 (1979).

[37] A. Stephan, J. Meijer, M. Hofert, H.H. Bukow, and C. Rolfs, Nucl Instrum Methods Phys Res, B 89, 420 (1994). 


\section{Figure captions}

Figure 1: Schematic of annular dark field (ADF) imaging. Scattered "dark field" electrons are collected to form the image. An image may be formed with "bright field" electrons, but this image includes unscattered background electrons which increase the current density without carrying image information.

Figure 2: Blur profile for the modeled optical system with $20 \mathrm{k}$ electrons at the equivalent density of $10^{6}$ electrons in $10 \mathrm{ps}$, fit to a Gaussian profile. The FWHM is $6.6 \mathrm{~nm}$, which is within the resolution limit given by shot noise.

Figure 3: (a) ADF image of a CdTe nanoparticle, tetrapod, and gold nanoparticle [32] and (b) a simulation of the same image assuming a scattered, detected fluence sufficient to satisfy the Rose criterion, and spatial smearing corresponding to the distribution shown in Figure 2. The image would be washed out on the scale of fig. 3(a) without ADF and relativistic electrons. 


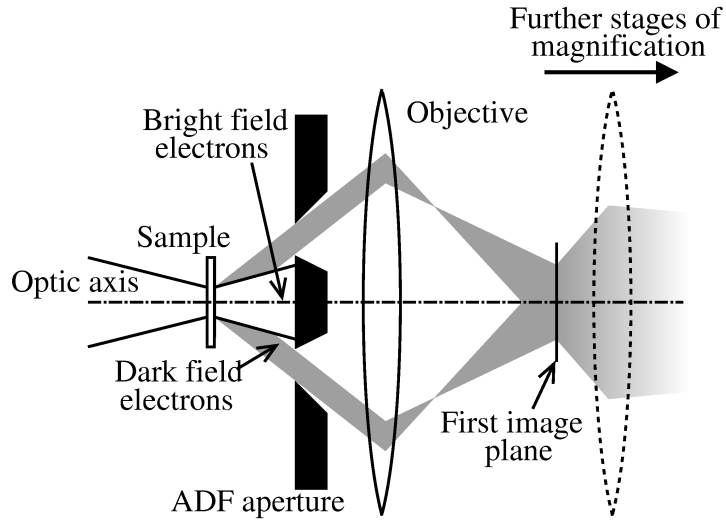

Figure 1: Schematic of annular dark field (ADF) imaging. Scattered "dark field" electrons are collected to form the image. An image may be formed with "bright field" electrons, but this image includes unscattered background electrons which increase the current density without carrying image information. 


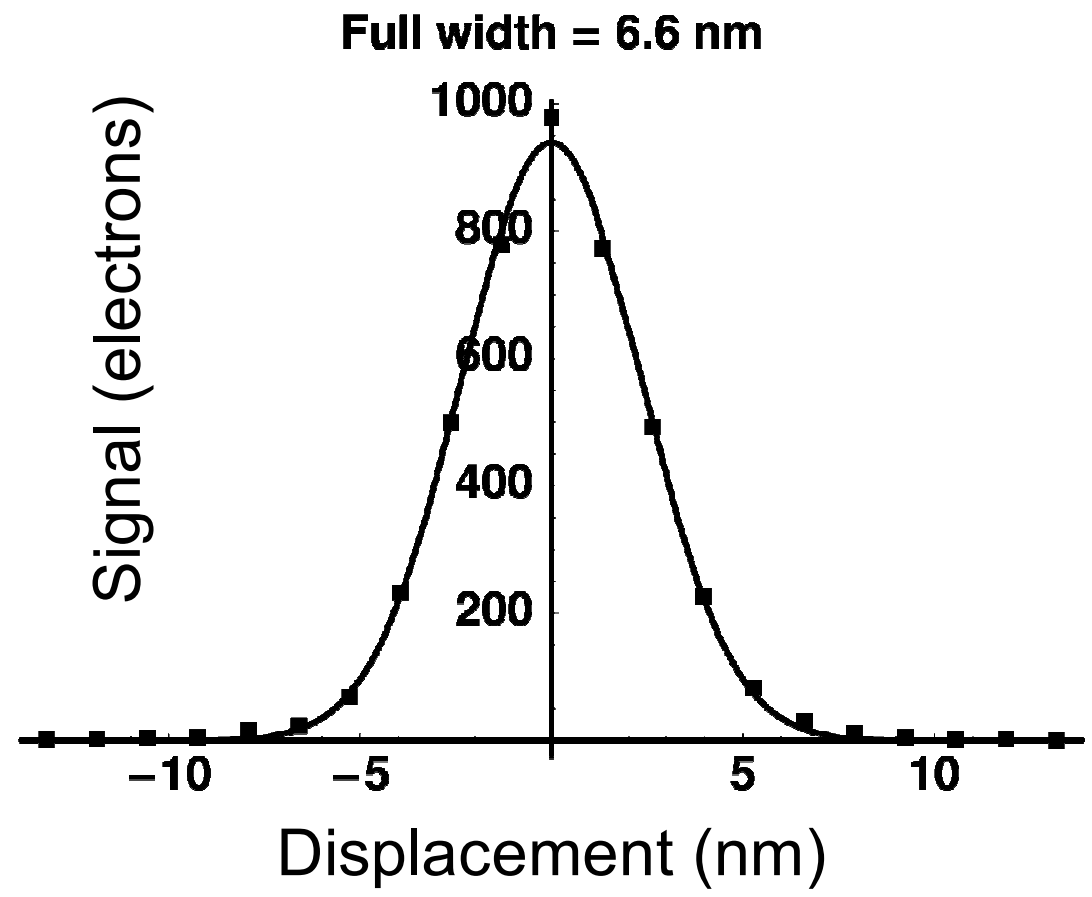

Figure 2: Blur profile for the modeled optical system with $20 \mathrm{k}$ electrons at the equivalent density of $10^{6}$ electrons in $10 \mathrm{ps,} \mathrm{fit} \mathrm{to} \mathrm{a} \mathrm{Gaussian} \mathrm{profile.} \mathrm{The} \mathrm{FWHM} \mathrm{is} 6.6 \mathrm{~nm}$, which is within the resolution limit given by shot noise. 


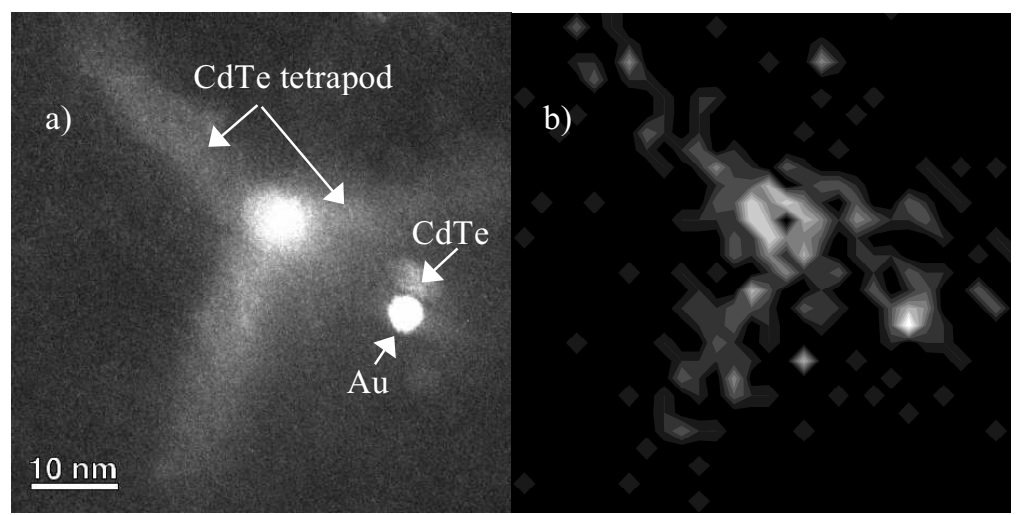

Figure 3: (a) ADF image of a CdTe nanoparticle, tetrapod, and gold nanoparticle [32] and (b) a simulation of the same image assuming a scattered, detected fluence sufficient to satisfy the Rose criterion, and spatial smearing corresponding to the distribution shown in Figure 2. The image would be washed out on the scale of fig. 3(a) without ADF and relativistic electrons. 